\title{
A rare case of humoral hypercalcemia of malignancy resulting from urothelial carcinoma
}

\author{
Jonathan Slusser ${ }^{1}$, Brian Beluch ${ }^{2}$ and Alan J Shienbaum ${ }^{3,4 *}$ \\ ${ }^{1}$ Department of Internal Medicine, School of Osteopathic Medicine, Rowan University, USA \\ ${ }^{2}$ Department of Endocrinology, Kennedy Health Alliance, USA \\ ${ }^{3}$ Department of Pathology, School of Osteopathic Medicine, Rowan University, USA \\ ${ }^{4}$ Department of Pathology, Kennedy University Hospital, USA
}

\begin{abstract}
Background: Humoral Hypercalcemia of malignancy is a common cause of hypercalcemia. This is typically associated with cancers of the head and neck and lung cancer.

Case presentation: We describe a 75 -year-old male with history of urothelial carcinoma since 2010 who presented with lethargy and acutely worsening weakness. His physical exam was unremarkable except for noted lethargy. Laboratory studies demonstrated elevated serum calcium with elevated serum PTHrP level. The patient's hypercalcemia responded to bisphosphonate therapy. Tissue from the prior transurethral resection of his bladder tumor demonstrated evidence of neuroendocrine differentiation by immunohistochemistry.
\end{abstract}

Conclusions: This represents a rare case of humoral hypercalcemia of malignancy resulting from recurrent urothelial carcinoma. Urothelial carcinoma is an uncommon cause of humoral hypercalcemia of malignancy and should be considered in the differential diagnosis of hypercalcemia in patients with a history of urothelial carcinoma or those at risk for this disease.

\section{Background}

Hypercalcemia of malignancy is a well-documented complication of cancer [1]. The mechanisms behind this are: 1) lytic destruction of bone by metastatic disease; or 2) humoral hypercalcemia with increased bone resorption and decreased renal clearance of calcium [2]. This is a unique case of recurrent urothelial carcinoma with associated hypercalcemia due to PTHrP secretion that manifested 6 years after initial diagnosis of urothelial carcinoma.

\section{Case presentation}

A 75-year-old male with past medical history of systolic heart failure, diabetes, hyperlipidemia, hypertension, diabetic neuropathy, CKD II with baseline creatinine $1.12 \mathrm{mg} / \mathrm{dL}$, history of urothelial carcinoma treated with chemotherapy and radiation, recurrent urinary tract infections, and chronic Foley catheter for urinary retention presented to emergency room with a one-month history of generalized weakness, fatigue and poor oral intake. He was initially diagnosed with high-grade invasive urothelial carcinoma in 2010 and had been following with urology for this issue. He had transurethral resections in 2014 and 2015 along with chemotherapy and radiation therapy with this last treatment occurring in November 2015. The patient never had issues with elevated calcium levels in the past.

When he presented to hospital with his current complaint, his serum calcium was elevated to $12.2 \mathrm{mg} / \mathrm{dL}$ with a serum albumin of $2.6 \mathrm{~g} / \mathrm{dL}$, resulting in corrected serum calcium of $13.3 \mathrm{mg} / \mathrm{dL}$. The patient was admitted to the hospital and treated with intravenous fluids overnight. Repeat calcium was $12.1 \mathrm{mg} / \mathrm{dL}$ the following morning. The patient was noted to be very lethargic on physical exam and cachectic- appearing. The patient and his family reported that he had not been eating well. He had also become bed-bound and unable to ambulate. He was only able to nod his head to answer questions. He was treated with Pamidronate $60 \mathrm{mg} I \mathrm{~V} \times 1$ dose over 8 hours.

An evaluation and work-up for the hypercalcemia was performed. Serum PTH level was suppressed at $6.1 \mathrm{pg} / \mathrm{mL}$ (normal range $12-88 \mathrm{pg}$ / $\mathrm{mL}$ ). His serum TSH level was 6.081. A skeletal radiographic survey was negative for any lytic disease. A chest $\mathrm{x}$-ray showed potential small nodularity in right lung base. A serum ACE level was $28 \mathrm{U} / \mathrm{L}$ (normal range 9-67 U/L). Serum Vitamin D 25 level was $20.5 \mathrm{ng} / \mathrm{mL}$ and a Vitamin D 1 level was $25<8 \mathrm{pg} / \mathrm{mL}$ (normal range $18-72 \mathrm{pg} / \mathrm{mL}$ ).

The patient had numerous CT abdomen studies done the most recent of which was three months prior showing no osseous structure abnormality, an enlarged prostate, chronic foley catheter and ureteral stents, and unchanged bladder wall thickening as compared to prior studies. A serum PTHrP level was found to be elevated at $147 \mathrm{pg} / \mathrm{mL}$ (normal range 14-27 pg/mL). He did suffer from acute kidney injury after infusion of pamidronate with serum creatinine increasing 1.39 $\mathrm{mg} / \mathrm{dL}$, up to $3.92 \mathrm{mg} / \mathrm{dl}$ on day 5 of his hospitalization. His kidney function did recover throughout his hospitalization with BUN 34 $\mathrm{mg} / \mathrm{dL}$ and creatinine $1.85 \mathrm{mg} / \mathrm{dL}$ on day 12 of the hospitalization.

Correspondence to: Alan J Shienbaum, DO, Department of Pathology, Kennedy University Hospital - Cherry Hill, 2201 Chapel Avenue West, Cherry Hill, NJ 08002, USA, Tel: 8564886560; Fax: 8564886624; E-mail: shienbaj@rowan.edu

Received: March 10, 2017; Accepted: March 22, 2017; Published: March 25, 2017 
Following treatment with Pamidronate, the serum calcium decreased to $8.5 \mathrm{mg} / \mathrm{dL}$, corresponding to a corrected serum calcium of $9.6 \mathrm{mg} / \mathrm{dL}$ within one week. The patient's overall status showed little improvement and thus he was evaluated by palliative care and was discharged home on hospice. The patient and family did not want to undergo any further diagnostic testing.

Histopathologic examination of the bladder tumor derived from the transurethral resection demonstrated a high-grade papillary urothelial carcinoma with invasion into muscularis propria (detrusor muscle). This tumor showed positive staining with p63 and high molecular weight cytokeratin immunohistochemical stains. In addition, neuroendocrine differentiation was demonstrated by a positive Neuron Specific Enolase (NSE) immunohistochemical stain (Figures 1 and 2).

Given the exclusion of other possible causative entities and the demonstration of neuroendocrine differentiation in this urothelial carcinoma, a diagnosis of humoral hypercalcemia of malignancy due to PTHrP secretion from urothelial carcinoma was established.

\section{Discussion}

Hypercalcemia of malignancy is a well-documented complication of cancer [1]. The mechanisms behind this are: 1) lytic destruction of bone by metastatic disease; or 2) humoral hypercalcemia with increased bone resorption and decreased renal clearance of calcium [2]. This is a unique case of recurrent urothelial carcinoma with associated hypercalcemia due to PTHrP secretion. No clinical or radiographic

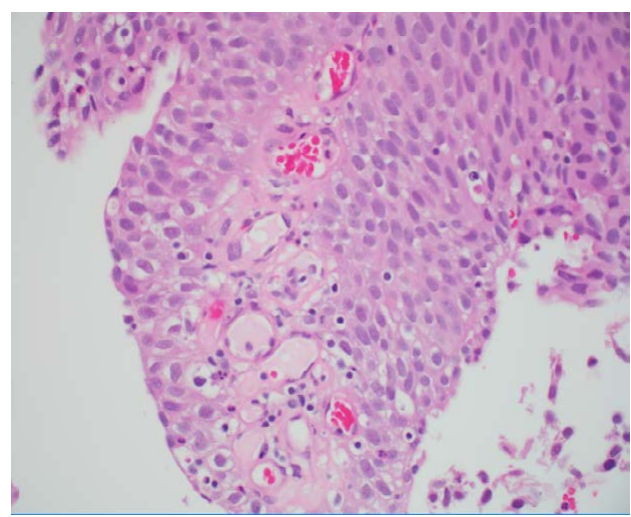

Figure 1. Hematoxylin- and eosin- stained section of this patient's urinary bladder tumor demonstrating a high-grade urothelial carcinoma with associated cytologic and architectural atypica (10x magnification).

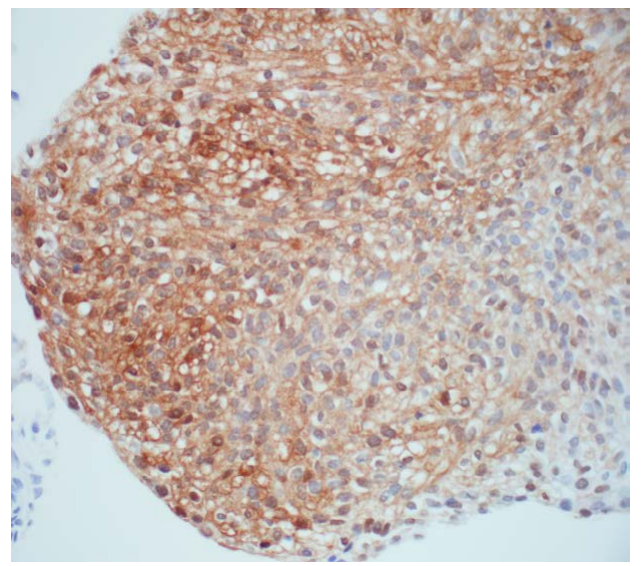

Figure 2. Neuron Specific Enolase (NSE) Immunohistochemistry stain of this tumor, which supports neuroendocrine differentiation (10x magnification). evidence of lytic destruction of bone by metastatic carcinoma was present in this case.

In the absence of metastatic disease, humoral hypercalcemia of malignancy is caused by the ectopic production of parathyroid hormone-related peptide (PTHrP) by tumor cells [2]. PTHrP is a hormone produced by cancer cells which has a 13-amino acid $\mathrm{N}$-terminal sequence homology with intact parathyroid hormone (PTH) [3]. The PTHrP binds to PTH receptors, which stimulates cAMP production and mimics the effects of PTH [3]. This leads to the inhibition of the action of osteoblasts and the stimulation of osteoclasts in the bone. The elaboration of PTHrP also promotes renal tubular calcium reabsorption, which further elevates serum calcium by inhibiting elimination [4]. Urothelial carcinoma is a reported but exceedingly rare cause of humoral hypercalcemia of malignancy [5].

In our patient's case, his urothelial carcinoma manifested years ago, but he did not have any clinically elevated calcium until recently. In our search through medical literature, there is only one other published case report showing recurrent urothelial carcinoma manifesting with hypercalcemia due to PTHrP secretion [6]. Other case reports have shown co-secretion with granulocyte colony-stimulating factor although our patient did not have any chronic elevations in his WBC, this was not directly measured $[7,8]$. Excess secretion of PTHrP should be considered in the differential diagnosis of hypercalcemia especially in the setting of malignancy. It is also worth considering the development of humoral hypercalcemia as a prognostic indicator. Although there have not been many patients develop PTHrP induced hypercalcemia from urothelial carcinoma, the mean survival time is 65 days (range 13-210 days); however, the sample size is limited with only six reported cases. Our patient had diagnosed urothelial cancer for several years but only recently developed hypercalcemia that was associated with significantly deteriorated status to the extent that hospice care was considered.

\section{Conclusion}

Humoral hypercalcemia of malignancy is a common cause of elevated calcium levels; however, urothelial carcinoma is an uncommon cause and should be considered in the differential diagnosis along with non-PTH mediated causes of hypercalcemia in patients with a history of urothelial carcinoma or those at risk for this disease.

\section{Declarations}

Consent for publication: Written informed consent was obtained from the patient for publication of this case report and any accompanying images. A copy of the written consent is available for review by the Editor-in-Chief of this journal.

\section{Ethics approval: Not applicable}

\section{Conflict of interest: None}

Data availability: Data sharing not applicable to this article as no datasets were generated or analyzed during the current study.

Funding sources: No funding sources were obtained for this case report.

Author contributions: JS helped draft the manuscript; $B B$ performed the medical work-up and participated in the design and coordination of this case report. AS help draft this manuscript, interpreted the pathology, took photographs of the pathology slides, and participated in the design and coordination of this case report. All 
authors read and approved the final manuscript.

\section{Acknowledgement: Not applicable.}

\section{References}

1. Jick S, Li L, Gastanaga V, Liede A (2015) Prevalence of Hypercalcemia of Malignancy Among Cancer Patients in the UK: Analysis of the Clinical Practice Research Data Link Database. Cancer Epidemiol 39: 901-907. [Crossref]

2. Williams RH, Larsen PR (2003) Williams Textbook of Endocrinology. 13th ed., Philadelphia, PA: Saunders, pp: 1265-1267, 1287-1288.

3. Jibrin IM, Lawrence GD, Miller CB (2006) Hypercalcemia of Malignancy in Hospitalized Patients. Hospital Physician 2006: 29-35.

4. Ratcliffe WA, Hutchesson AC, Bundred NJ, Ratcliffe JG (1992) Role of assays for parathyroid-hormone-related protein in investigation of hypercalcaemia. Lancet 339 164-167. [Crossref]

5. Szymanski J, Zaher O, Khushbu P, Mitchell S (2016) Incidence of Humoral Hypercalcemia of Malignancy Among Hypercalcemic Patients with Cancer. Clinica Chimica Acta 190-193.

6. Sullivan EE (2014) BMJ case reports: A rare complication of transitional cell carcinoma of the renal pelvis: parathyroid hormone-related peptide-induced hypercalcaemia. $B M J$ Publishing Group.

7. Hirasawa K, Kitamura T, Oka T, Matsushita H (2002) Bladder tumor producing granulocyte colony-stimulating factor and parathyroid hormone related protein. $J$ Urol 167: 2130. [Crossref]

8. Tsuchiya F, Ikeda I, Kanda F, Fukuoka H (2001) A case of bladder tumor producing granulocyte-colony stimulation factor and parathyroid hormone-related protein. Hinyokika Kiyo 47: 873-876. [Crossref]

Copyright: (C2017 Slusser J. This is an open-access article distributed under the terms of the Creative Commons Attribution License, which permits unrestricted use, distribution, and reproduction in any medium, provided the original author and source are credited. 